Z Gerontol Geriat 2017 · 50:395-396 DOI 10.1007/s00391-017-1273-8

Online publiziert: 14. Juni 2017

(c) Springer Medizin Verlag GmbH 2017

CrossMark

\title{
Frank Oswald ${ }^{1}$. Annette Franke ${ }^{2}$. Eva-Marie Kessler ${ }^{3}$
}

'Interdisziplinäre Alternswissenschaft (IAW), Fachbereich Erziehungswissenschaften, Frankfurter Forum für interdisziplinäre Alternsforschung (FFIA), Goethe-Universität Frankfurt am Main, Frankfurt am Main, Deutschland

${ }^{2}$ Gesundheitsförderung und Methoden und Konzepte der Sozialen Arbeit, Fachbereich Soziale Arbeit, Evangelische Hochschule Ludwigsburg/Protestant University of Applied Sciences, Ludwigsburg, Deutschland

${ }^{3}$ Gerontopsychologie, Fakultät für Naturwissenschaften, MSB Medical School Berlin/Hochschule für Gesundheit und Medizin, Siemens Villa, Berlin, Deutschland

\section{Beiträge zum Themenschwer- punkt aus der Sektion III (Sozial- und verhaltenswissenschaftliche Gerontologie) der Deutschen Gesellschaft für Gerontologie und Geriatrie e.V. (DGGG)}

Gerontologie ist eine Wissenschaft mit vielen Teilbereichen und Anwendungsbezügen. Die sozial- und verhaltenswissenschaftliche Gerontologie untersucht die Person bzw. soziales Handeln im Kontext sozialer Beziehungen und gesellschaftlichen, also beispielsweise politischen, historischen und kulturellen Bedingungen, Institutionen und Rollen. Sowohl Person- als auch Kontextfaktoren können zu Kontinuität oder Veränderung beitragen. Seitens der Person werden beispielsweise Lebensstile, kognitive Fähigkeiten, Gesundheit und Erfahrungen im Lebenslauf adressiert. Als Kontexte gelten neben den sozialen auch räumliche Umwelten, materielle Ressourcen und soziale Ungleichheit ebenso wie gesellschaftliche Altersbilder und soziopolitische Rahmenbedingungen. Beiträge aus zahlreichen Fachdisziplinen, die sich mit diesen Fragestellungen beschäftigen (z.B. Psychologie, Soziologie, Gesellschaftswissenschaften, Erziehungswissenschaften, Rechtswissenschaft, Wirtschaftswissenschaften, Gesundheits- und Therapiewissenschaften) ebenso wie zunehmend interdisziplinäre Ansätze bereichern und prägen die gerontologische Forschung in Deutschland. Neben der Forschung ist gleichermaßen die wissenschaftlich fundierte gerontologische Ausbildung in den vielfältigen sozial- und verhaltenswissenschaftlichen Bereichen von Bedeutung. Und schließlich richtet eine sowohl grundlagen- als auch anwendungsorientierte gerontologische Forschung stets den Blick auf die Folgen für die wissenschaftliche Politikberatung.

Das Jubiläum der Zeitschrift für Gerontologie und Geriatrie bietet uns einen willkommenen Anlass, die letzten 50 Jahre gerontologischer Ausbildung, Forschung und Politikberatung Revue passieren zu lassen, um daraus neue Aufgaben und Herausforderungen für die Zukunft abzuleiten. Selbstverständlich kann dies keine erschöpfende Rundumschau sein, sondern vielmehr ein „dreigeteilter“ ausgewählter Blick, der auch die jeweiligen Sichtweisen der Autor/ -innen widerspiegelt.

Im ersten Beitrag von Eva-Marie Kessler, Andreas Hoff und Annette Franke zum Thema „Gerontologisch orientierte Studiengänge in Deutschland - eine kritische Bestandsaufnahme" werden aktuel- 
le Studienangebote in Deutschland beschrieben und einer kritischen Reflexion unterzogen. Der Beitrag basiert auf Anregungen des Arbeitskreises „Gerontologische Aus- und Weiterbildung " der Deutschen Gesellschaft für Gerontologie und Geriatrie (DGGG). Neben einer diagnostizierten Zunahme von Angeboten innerhalb der letzten 10 Jahre identifizieren die Autor/-innen heterogene Standortdynamiken in dieser Zeit. So findet ein Wandel von expliziten Gerontologie-Studiengängen hin zu Studiengängen statt, die in anderen Disziplinen verwurzelt sind (Soziologie, Soziale Arbeit, Psychologie, Sportwissenschaft, Gesundheits- bzw. Therapiewissenschaften).

Der zweite Beitrag von Clemens TeschRömer, Hans-Werner Wahl und Martina Brandt trägt den Titel „Gerontologische Forschung im Bereich der Sozial- und Verhaltenswissenschaften in Deutschland seit 1968: Rückblick, kritische Würdigung und Perspektiven“. Die Autor/ -innen haben die sozial- und verhaltenswissenschaftliche Alternsforschung der letzten 50 Jahren in Deutschland aufgearbeitet und vertreten die Thesen, dass (1) der Aufbau der diesbezüglichen Forschung in den 1960er- und 1970erJahren langwierig war, dass (2) die Bedeutung dieser Forschung in den 1980erund 1990er-Jahren wuchs und (3) die Strahlkraft der sozial- und verhaltenswissenschaftlichen Alternsforschung in den letzten 20 Jahren zunahm, sowie dass (4) insbesondere die methodische Qualität diesbezüglicher Forschung (am Beispiel von Survey-Daten) in Deutschland hoch und international sichtbar ist.

Im dritten Beitrag beschäftigen sich schließlich Andreas Kruse, Adelheid Kuhlmey und Gerhard Naegele mit „Gerontologischer Politikberatung - Möglichkeiten und Grenzen wissenschaftlicher Beratung“. Dabei wenden sie sich zunächst dem Verständnis von Politikberatung zu, das im Spannungsfeld zwischen Funktionen der Aufklärung und Kritik und den daraus ableitbaren Beratungsmodellen (technokratisch, dezisionistisch, pragmatisch) entwickelt wird. Danach wird sozialwissenschaftliche Politikberatung u.a. anhand der Beispiele der Enquete-Kommission Demo- grafischer Wandel und den mittlerweile sieben Altenberichten der Bundesregierung erläutert.

Die Beiträge ermöglichen einen fundierten Einblick in aktuelle und relevante Themen deutscher sozial- und verhaltenswissenschaftlicher Gerontologie der letzten Jahrzehnte, auch wenn deren Facettenreichtum nicht angemessen und in aller Fülle abgebildet werden kann. Das heißt auch: Eine sozial- und verhaltenswissenschaftliche Ausbildung, Forschung und Politikberatung ist nie abgeschlossen und bleibt auf die Zukunft hin gestaltbar. Dies gilt beispielsweise für die Stärkung einer angemessenen Forschungsinfrastruktur ebenso wie für die Frage nach Kernelementen gerontologischer Studienangebote. Wenn dieser Versuch einer ausgewählten kleinen Bestandsaufnahme die Leser/-innen also dazu anregt, Gerontologie in Zukunft selbst mitgestalten zu wollen, haben wir unser Ziel erreicht.

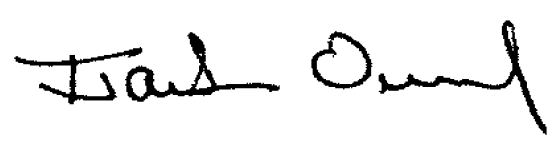

Frank Oswald

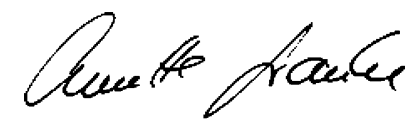

Annette Franke

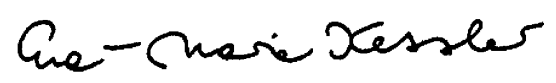

Eva-Maria Kessler

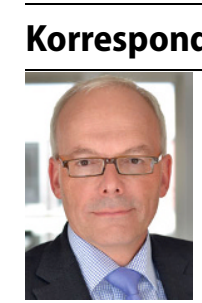

Korrespondenzadresse

Prof. Dr. F. Oswald

Interdisziplinäre Alternswissenschaft (IAW), Fachbereich

Erziehungswissenschaften, Frankfurter Forum für inter-

disziplinäre Alternsforschung (FFIA), Goethe-Universität Frankfurt am Main Theodor-W.-Adorno-Platz 6 60323 Frankfurt am Main,

Deutschland

oswald@em.uni-frankfurt.de

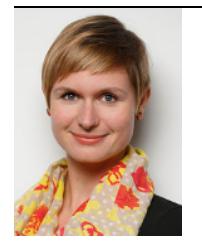

Prof. Dr. A. Franke

Gesundheitsförderung und Methoden und Konzepte der Sozialen Arbeit, Fachbereich Soziale Arbeit, Evangelische Hochschule Ludwigsburg/ Protestant University of Applied Sciences

Paulusweg 6, 71638 Ludwigsburg, Deutschland A.Franke@eh-ludwigsburg.de

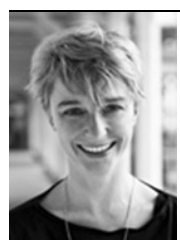

Prof. Dr. habil. E.-M. Kessler Gerontopsychologie, Fakultät für Naturwissenschaften, MSB Medical School Berlin/ Hochschule für Gesundheit und Medizin, Siemens Villa Calandrellistrasse 1-9, 12247 Berlin, Deutschland eva-marie.kessler@medicalschoolberlin.de

Interessenkonflikt. F. Oswald, A. Franke und E.-M. Kessler geben an, dass kein Interessenkonflikt besteht. 